\title{
Particle Physics from D-Branes at Gepner Points
}

\author{
Gerardo Aldazabal* \\ Instituto Balseiro, Centro Atómico Bariloche, Argentina \\ E-mail: aldazaba@cab.cnea.gov.ar \\ E. Andrés \\ Instituto Balseiro, Argentina \\ E-mail: andres@cab. cnea.gov.ar

\section{J. E. Juknevich} \\ Department of Physics and Astronomy, Rutgers University, \\ Piscataway, NJ 08855 USA \\ E-mail: juknevij@physics.rutgers.edu
}

We discuss the building of Standard-like models from configurations of stacks of orientifold planes and D-branes on an internal space with the structure $(\text { Gepner model })^{c=3 n} \times \mathbf{T}^{\mathbf{2}(\mathbf{3}-\mathbf{n})} / \mathbf{Z}_{\mathbf{N}}$, for $n=1,2,3$. In particular we concentrate in the, interesting, $n=2$ case, which would correspond to orbifolds of a $K 3$ six dimensional manifold times a two dimensional torus.

Such constructions are performed in two steps. In a first step, we consider $D=6$ Type II B orientifolds on Gepner points, in the diagonal invariant case and for both, odd and even, affine levels. We build up the explicit expressions for B-type boundary states and crosscaps and obtain the amplitudes among them. From such amplitudes we read the corresponding spectra and the tadpole cancellation equations. In a second step we further compactify on a $\mathbf{T}^{\mathbf{2}}$ torus, by simultaneously orbifolding the Gepner and the torus internal sectors. The embedding of the orbifold action in the brane sector breaks the original gauge groups and leads to $\mathscr{N}=1$ supersymmetric chiral spectra. Whenever even orbifold action on the torus is considered, new branes, with worldvolume transverse to torus coordinates, must be included. Interestingly enough, large transverse extra dimensions can be used to lower the string scale.

As an illustration we present a 3 generations Left-Right symmetric model that can be further broken to a MSSM model.

Fifth International Conference on Mathematical Methods in Physics

24 - 28, April 2006

Rio de Janeiro, Brazil

\footnotetext{
${ }^{*}$ Speaker.
} 


\section{Introduction}

The quest of the Standard Model like vacua, from open string interacting conformal field theories, received considerable attention in last years. In particular, much progress has been achieved in the context of orientifolds of Type II string compactified on Gepner models [1, 2, 3, 4, 5, 6, 7] Gepner models [8] are special points of Calabi Yau manifolds, at string scale, that allow for a description in terms of an exactly solvable rational CFT. Preliminary works in heterotic string theories and Type II orientifolds on Gepner points were presented [9, 10].

The rules for computing spectra and the tadpole cancellation equations have been derived for generic situations. Nevertheless, even if concise and rather simple generic expressions can be obtained, the computation of spectra for specific models can become rather cumbersome due to the, generically, huge number of open string states involved. Only solving the tadpole consistency equations can represent a difficult task even for a fast computer. Therefore a systematics is needed in order to be able to extract any useful information. In this sense a remarkable computing search for models with Sandard like model spectra was performed in [5] by restricting the scan to four stacks of SM branes, by following the ideas advanced in [11] in the context of intersecting brane models [13] on toroidal like manifolds. In fact, thousands of SM like models were found. It is worth mentioning that even the simplest of these models requires to introduce a huge number of projections and to solve several tadpole equations. In Ref. [6] a hybrid Type IIB orientifold construction was proposed where the internal sector is built up from a Gepner sector times a torus. By choosing a torus invariant under some of the known $\mathbf{Z}_{\mathbf{N}}$ phase symmetries of Gepner models, an orbifold by such symmetries was then performed. Thus, schematically, in $D=4+2 n$ the internal sector is given by (Gepner model) $)^{c_{\text {int }}=9-3 n} \times \mathbf{T}^{\mathbf{2 n}} / \mathbf{Z}_{\mathbf{N}}$ (where $c_{\text {int }}$ is the internal central charge). The orbifold action is simultaneously embedded as a twist on Chan Paton factors on the open string sector resulting in a breaking of the starting $D$ dimensional Gepner orientifold gauge groups. In particular, such constructions lead to $\mathscr{N}=1 D=4$ chiral models. Further elaboration on this idea was advanced in Ref. [7].

Hybrid Gepner-torus models have some interesting features. An important, practical, observation [6] is that the number of Gepner models (see [10]) involved, 3 in $D=8$ or 16 in $D=6$, is remarkably lower than the 168 models in $D=4$ (without including moddings) and so it is the number of internal states. Also, many features can be studied analytically without the need of computers. From the phenomenological point of view, the possibility of having large extra dimensions, in the torus directions, could be an appealing feature allowing for some control over the string scale.

In this talk we discuss this hybrid models proposal, mainly based on the work of Ref. [7], where the internal sector is built up from $D=6$ Gepner models times a two torus. From expressions for B-type boundary states and crosscaps we read the tadpole cancellation equations and the rules for reading the spectra. An explicit example (the 6620 model) is developed in detail. We further compactify on a $\mathbf{T}^{\mathbf{2}}$ torus by simultaneously orbifolding the Gepner and the torus internal sectors and by embedding the orbifold action on the brane sector. Detailed rules for obtaining the $D=4$ model spectra and tadpole equations are shown. We conclude with an illustration of how to obtain a 3 generations Left-Right symmetric model (which can be further broken into a MSSM model) from a $Z_{4}$ orbifold of the, $D=6,6620$ diagonal Gepner model times a torus. 


\section{Type II orientifolds, crosscaps and boundary states}

Essentially, an orientifold model is obtained by dividing out the orientation reversal symmetry of Type II string theory (see for instance [15, 3]). Schematically, Type IIB torus partition function is defined as

$$
\mathscr{Z}_{T}(\tau, \bar{\tau})=\sum_{a, b} \chi_{a}(\tau) \mathscr{N}^{a b} \bar{\chi}_{b}(\bar{\tau})
$$

where the characters $\chi_{a}(\tau)=\operatorname{Tr}_{\mathscr{C} a} q^{L_{0}-\frac{c}{24}}$, with $q=e^{2 i \pi \tau}$, span a representation of the modular group of the torus generated by $\mathrm{S}: \tau \rightarrow-\frac{1}{\tau}$ and $\mathrm{T}: \tau \rightarrow \tau+1$ transformations. $\mathscr{H}_{a}$ is the Hilbert space of a conformal field theory with central charge $c=15$, generated from a conformal primary state $\phi_{a}$ (similarly for the right moving algebra). In particular $\chi_{a}\left(-\frac{1}{\tau}\right)=S_{a a^{\prime}} \chi_{a^{\prime}}(\tau)$ and modular invariance require $S \mathscr{N} S^{-1}=\mathscr{N}$ (for left -right symmetric theories $\mathscr{N}^{a b}=\mathscr{N}^{b a}$ ). Generically, the characters can be split into a spacetime piece, contributing with $c_{s t}=\bar{c}_{s t}=\frac{3}{2} D$ and an internal sector with $c_{\text {int }}=\bar{c}_{\text {int }}=\frac{3}{2}(10-D)$.

Let $\Omega$ be the reversing order (orientifolding) operator permuting right and left movers. Modding by order reversal symmetry is then implemented by introducing the projection operator $\frac{1}{2}(1+$ $\Omega$ ) into the torus partition function. The resulting vacuum amplitude reads

$$
\mathscr{Z}_{\Omega}(\tau, \bar{\tau})=\mathscr{Z}_{T}(\tau, \bar{\tau})+\mathscr{Z}_{K}(\tau-\bar{\tau})
$$

The first term is just the symmetrization (or anti-symmetrization in case states anticommute) of left and right sector contributions indicating that two states differing in a left-right ordering must be counted only once. The second term is the Klein bottle contribution and takes into account states that are exactly the same in both sectors. In such case, the operator $e^{2 i \pi \tau L_{0}} e^{-2 i \pi \bar{\tau} \bar{L}_{0}}$, when acting on the same states, becomes $e^{2 i \pi 2 i t_{K} L_{0}}$ with $\tau-\bar{\tau}=2 i t_{K}$ and thus

$$
\mathscr{Z}_{K}\left(2 i t_{K}\right)=\frac{1}{2} \sum_{a} \mathscr{K}^{a} \chi_{a}\left(2 i t_{K}\right)
$$

where $\left|\mathscr{K}^{a}\right|=\mathscr{N}^{a a}$. The Klein bottle amplitude in the transverse channel is obtained by performing an $\mathrm{S}$ modular transformation such that

$$
\tilde{\mathscr{Z}}_{K}(i l)=\frac{1}{2} \sum_{a} O_{a}^{2} \chi_{a}(i l)
$$

with $l=\frac{1}{2 t_{K}}$ and

$$
\left(O^{a}\right)^{2}=2^{D} \mathscr{K}^{b} S_{b a}
$$

This notation for the closed channel coefficients highlights the fact that the Klein bottle transverse channel represents a closed string propagating between two crosscaps (orientifold planes) states. Namely, a quantum state $|C\rangle$, describing the crosscap can be found such that the KB amplitude can be expressed as

$$
\tilde{\mathscr{Z}}_{K}(i l)=\frac{1}{2}\left\langle C\left|q^{\frac{1}{2} H_{c l}}\right| C\right\rangle .
$$

with $H_{c l}=L_{0}-\tilde{L}_{0}-\frac{c}{12}$. 
Indeed, crosscap states can be formally expanded in terms of Ishibashi states [16] such that

$$
\left.|C\rangle=O_{a}|a\rangle\right\rangle_{C}
$$

with

$$
{ }_{C}\left\langle\left\langle b\left|q^{\frac{1}{2} H_{c l}}\right| a\right\rangle\right\rangle_{C}=\delta_{a, b} \chi^{a}(\tilde{q})
$$

and $\tilde{q}=e^{2 i \pi l}$.

When integrated over the tube length, such amplitude leads, for massless states, to tadpole like divergences. In particular, for RR massless states, such tadpoles must be cancelled for the theory to be consistent. Notice that, for such fields, $O_{a}$ represents the charge of the orientifold plane (crosscap) under them and, therefore, inclusion of an open string sector with D-branes carrying $-O_{a}$ RR charge provides a way for having a consistent theory [17] with net vanishing charge.

Therefore, we introduce stacks of boundary states $|\alpha\rangle$ (referred to as "brane- $\alpha$ ")

$$
\left.|\alpha\rangle=\sum_{a} D_{\alpha}^{a}|a\rangle\right\rangle_{B}
$$

such that the amplitude, describing propagation of strings between "intersecting" stacks $\alpha$ and $\beta$ can be written as

$$
\tilde{\mathscr{Z}}_{\beta, \alpha}(i l)=\left\langle\beta\left|q^{\frac{1}{2} H_{c l}}\right| \alpha\right\rangle=\sum_{a} D_{\alpha}^{a} D_{\beta}^{a} \chi^{a}(l)=\sum_{b} C_{\beta, \alpha}^{b} \chi^{b}(t / 2)
$$

where in the last step we have perform an $S$ modular transformation to direct channel.

Here

$$
C_{\beta, \alpha}^{b}=\sum_{a} D_{\beta}^{a} D_{\alpha}^{a} S_{a b}
$$

is the multiplicity of open string states contained in $\chi_{a}$. Namely, it counts open string sector states of the form

$$
\left|\Phi_{a} ; \beta, \alpha\right\rangle
$$

where $\Phi_{a}$ is a world sheet conformal field and $\alpha, \beta$ label the type of "branes" where the string endpoints must be attached to. $C_{\beta, \alpha}^{a}$ are positive integers (actually $C_{\beta, \alpha}^{a}=0,1,2$ [3]) generated when the trace over open states $\left|\Phi_{a} ; \beta, \alpha\right\rangle$ is computed.

The full cylinder partition function is obtained when summing over all possible stacks of $n_{\alpha}$ branes, namely

$$
\tilde{\mathscr{Z}}_{C}(i l)=\sum_{a} D_{a}^{2} \chi^{a}(l)
$$

with $D^{a}=\sum_{\alpha} n_{\alpha} D_{\alpha}^{a}$.

In a similar manner, strings propagating between branes and orbifold planes give rise to Möbius strip amplitude

$$
\tilde{\mathscr{Z}}_{M}(i l)=2 D_{a} O_{a} \hat{\chi}^{a}\left(l+\frac{1}{2}\right)
$$

By modular transforming to direct channel we obtain multiplicities of open string states between a brane and its orientifold image

$$
O_{a}\left(n_{\alpha} D_{\alpha}^{b}\right) P_{b a}=\mathscr{M}_{a}=M_{\alpha}^{b} n_{\alpha}
$$


where we have used the fact that characters in the direct and transverse channels of the Möbius strip are related by the transformation [18] P: $i t_{M}+\frac{1}{2} \rightarrow \frac{i}{4 t_{M}}+\frac{1}{2}$ generated from the modular transformations $\mathrm{S}$ and $\mathrm{T}$ as $\mathrm{P}=\mathrm{TST}^{2} \mathrm{~S}$.

For indices $a$ representing massless RR fields $D_{a}$ is the D-brane RR charge. Therefore zero net RR charge requires the

$$
O_{a}+n_{\alpha} D_{\alpha}^{a}=0
$$

tadpole cancellation equations.

\section{Orientifolds of $D=6$ Gepner models}

In Gepner models [8], in $D$ space time dimensions, the internal sector is given by a tensor product of $r$ copies of $N=2$ superconformal minimal models with levels $k_{j}, j=1, \ldots, r$ and central charge

$$
c_{j}=\frac{3 k_{j}}{k_{j}+2} \quad, \quad k_{j}=1,2, \ldots
$$

such that internal central charge $c_{i n t}=\sum_{j=1}^{r} c_{j}^{i n t}=12-3(D-2) / 2$.

Unitary representations of $N=2$ minimal models are encoded in primary fields labelled by three integers $(l, m, s)$ such that $l=0,1, \ldots, k ; l+m+s=0 \bmod 2$. These fields belong to the NS or R sector when $l+m$ is even or odd respectively The primary field information of the complete theory can be conveniently collected in the vectors $\lambda$ and $\mu$ [7]. Thus, the index $a$ in the previous section amounts here for $a=(\lambda, \mu)$ in Gepner's case. Spacetime supersymmetry and modular invariance are implemented by keeping in the spectrum only states for which the total $U(1)$ charge is an odd integer.

In six dimensions $c_{i n t}=6$ and 16 different possible Gepner models exist, which are associated to $K 3$ surfaces [10, 19] i.e. $\bar{k}=(1,1,1,1,1,1), \quad \bar{k}=(2,2,2,2), \quad \bar{k}=(0,2,6,6)$, etc.

Notice that, in some cases, $k=0$ blocks have been added. Even if such terms are irrelevant in a closed string theory (for instance the central charge remains invariant), they have been shown to have a non trivial (K-theory) effect when open string sector is included. In fact, an even (odd) number of internal minimal blocks is required (see for instance $[4,14])$ in $D=6(D=4)$ for consistency. Actually, for the sake of simplicity we will consider the case where the internal sector is a tensor product of $r=6$ conformal blocks. This will allow us to simultaneously consider cases with $3,4,5$, and 6 conformal blocks such as $(6)^{2}(2),(2)^{4}, 1^{4}$ or $(1)^{6}$ by adding, if necessary, conformal blocks with level $k=0$.

The Klein bottle amplitude is determined from that of the torus up to signs representing different ways of "dressing" the world-sheet parity $\Omega[6,2])$. We denote the dressed orientifold operator as $\Omega_{\Delta, \omega_{j}}^{B}$.

From the Klein bottle amplitude in the transverse channel we can read the expression for the crosscap state up to signs that can be fixed from the Möbius strip amplitude. The result is that the crosscap state is given by ${ }^{1}$

\footnotetext{
${ }^{1}$ For explicit expressions for modular matrices $P_{l^{\prime}, l}$ and $S_{l^{\prime}, l}$ see $[6,2]$.
} 


$$
\begin{aligned}
|C\rangle_{B}^{N S}= & \frac{1}{\kappa_{c}} \sum_{\lambda^{\prime}, \mu^{\prime}}{ }^{e v} \sum_{v_{0}=0}^{\frac{K}{2}-1} \sum_{v_{j}, \tilde{\tilde{v}}_{1}=0}^{1} \sum_{\varepsilon_{j}=0}^{1}\left(\prod_{k<l}(-1)^{v_{k} v_{l}}\right)(-1)^{v_{0}}(-1)^{\sum_{j} v_{j}} \\
& (-1)^{\sum \omega_{j} \Lambda_{0}^{\prime} / 2} e^{2 \pi i v_{0} \sum \frac{\Delta_{j}}{k_{j}+2}} \delta_{\Lambda_{0}^{\prime}, 2+2 v_{0}+2 \tilde{v}_{1}+2 \sum v_{j}+2 \sum \omega_{j}}^{(4)} \delta_{\Lambda_{1}^{\prime}, 2 v_{0}+2 \tilde{v}_{1}}^{(4)} \\
& \left.\prod_{j=1}^{r}\left(\sigma_{\left(l_{j}^{\prime}, m_{j}^{\prime}, s_{j}^{\prime}\right)} \frac{P_{l_{j}^{\prime}, \varepsilon_{j} k_{j}}}{\sqrt{S_{l_{j}^{\prime}, 0}}} \delta_{m_{j}^{\prime}, 2 v_{0}+\left(1-\varepsilon_{j}+\omega_{j}\right)\left(k_{j}+2\right)}^{\left(2 k_{j}+4\right)} \delta_{s_{j}^{\prime}, 2 v_{0}+2 v_{j}+2\left(1-\varepsilon_{j}\right)}^{(4)}(-1)^{\varepsilon_{j} \frac{\left(m_{j}^{\prime}+s_{j}^{\prime}\right)}{2}}\right)\left|\lambda^{\prime}, \mu^{\prime}\right\rangle\right\rangle_{c} \mid
\end{aligned}
$$

The normalization is chosen so that the overlap of the crosscap with itself yields the transverse Klein amplitude 2.6. Here

$$
\Delta=H \sum_{i=1}^{r} \frac{\Delta_{i}}{k_{i}+2}, \quad \omega=\sum_{i=1}^{r} \omega_{i}
$$

with $H=\operatorname{lcm}\left\{k_{i}+2\right\}, \quad K=\operatorname{lcm}\left(4,2 k_{j}+4\right)$ and $\Delta, \omega_{i}$ denote the "dressings".

In order to cancel tadpole-like divergences, boundary states must be introduced. We consider the B-type RS-boundary states [20]

$$
\begin{aligned}
|\alpha\rangle_{B}= & \left|S_{0}, \tilde{S}_{1} ;\left(L_{j}, M_{j}, S_{j}\right)_{j=1}^{r}\right\rangle_{B}=\frac{1}{\kappa_{\alpha}^{B}} \sum_{\lambda^{\prime}, \mu^{\prime}}^{\beta, b}(-1)^{\frac{\Lambda_{0}^{2}}{2}} e^{-i \pi \Lambda_{0}^{\prime} \frac{S_{0}}{2}} e^{-i \pi \Lambda_{1}^{\prime} \frac{\tilde{S}_{1}}{2}} \\
& \left.\prod_{j=1}^{r} \frac{S_{l_{j}^{\prime}, L_{j}}}{\sqrt{S_{l_{j}^{\prime}, 0}}} e^{i \pi \frac{m_{j}^{\prime} M_{j}}{k_{j}+2}} e^{-i \pi \frac{s_{j}^{\prime} S_{j}}{2}}\left|\lambda^{\prime}, \mu^{\prime}\right\rangle\right\rangle
\end{aligned}
$$

where "b" in the summatory indicates that $m_{j}=b \bmod \left(k_{j}+2\right)$. In fact, due to supersymmetry and field identifications these B-type boundary states only depend on $\mathbf{L}=\left(L_{1}, \ldots, L_{r}\right)$ with $L_{i} \leq k_{i} / 2$, $M=H \sum \frac{M_{i}}{k_{i}+2}$ and $S=\sum S_{i}$. However, whenever a label $L_{i}$ reaches $k_{i} / 2$, extra copies of the gauge field may appear propagating on the brane world-volume. In this case, it is necessary to resolve the branes into elementary branes such that only a single gauge field is propagating on the worldvolume. The details depend on the values of $|\mathbf{S}|$ counting the number of $i$ such that $L_{i}=k_{i} / 2$. It can be shown [4] that when $|\mathbf{S}|$ is an odd integer the elementary branes are given by

$$
\left|\alpha_{e l e}\right\rangle_{B}=\frac{1}{2^{\frac{|S|-1}{2}}}|\alpha\rangle_{B}
$$

Instead, if $|\mathbf{S}|$ is even there is an extra $\mathbf{Z}_{2}$-valued label $\psi$ taking values \pm so that the elementary boundary states are now labelled by $\mathbf{L}, \mathbf{M}, \mathbf{S}, \psi$. The original boundary states can be written in terms of the elementary ones as

$$
|\alpha\rangle_{B}=\frac{1}{2^{|\mathbf{S}|}}\left\{|\alpha+\rangle_{B}+|\alpha-\rangle_{B}\right\}
$$

where $\alpha$ stands for all labels different from $\psi$. The two boundary states $|\alpha \pm\rangle_{B}$ contain new states from the twisted $(c, c)$ RR sector. Explicit expressions for such states are given in [7].

The massless fields in the $6 \mathrm{D}$ spacetime theory are the vector field $(2,0)(0,0,0)^{6}$ and the hypermultiplets $(0,0) \prod_{j}\left(l_{j}, l_{j}, 0\right)$ with $\sum_{j} \frac{l_{j}}{k_{j}+2}=1$. They are contained in the cylinder and Möbius 
amplitude which we present next. The bosonic and massless part of the cylinder amplitude between two D-branes $|\mathbf{L}, M\rangle$ and $\left|\mathbf{L}^{\prime}, M^{\prime}\right\rangle$ is generally given by

$$
\frac{1}{2} \frac{1}{N_{\mathbf{S}}} \frac{1}{N_{\mathbf{S}^{\prime}}} \sum_{\lambda, \mu}^{s r} \sum_{\varepsilon_{1}, \ldots, \varepsilon_{r}=0}^{1}\left(\prod_{i=1}^{r} N_{L_{j}, L_{j}^{\prime}}^{\left|\varepsilon_{j} k_{j}-l_{j}\right|}\right) \delta_{\sum_{i} \varepsilon_{i}=1+\frac{s_{0}}{2}}^{(2)} \chi_{\mu}^{\lambda}
$$

where $N_{L_{1}, L_{2}}^{l}$ are the $S U(2)$ fusion coefficients ( $\left.[21,22]\right)$ and

$$
N_{\mathbf{S}}= \begin{cases}2^{|\mathbf{S}| / 2} & \text { if }|\mathbf{S}| \text { even } \\ 2^{[|\mathbf{S}|-1] / 2} & \text { if }|\mathbf{S}| \text { odd }\end{cases}
$$

eliminates any extra counting when some of the D-branes are short. Besides, we have defined an extra label $s_{0}=\Lambda_{0}+\Lambda_{1} \bmod 4$ (see Appendix A) taking the values 0,2 whenever the fields are in the scalar and vector representations, respectively (Note that in six dimensional spacetime $s_{0}=0,2$ also for the spinor representations, so this definition strictly makes sense when we restrict ourselves to bosonic representations). When the amplitude between two short-orbit branes $|\mathbf{L}, \psi\rangle$ and $\left|\mathbf{L}^{\prime}, \psi^{\prime}\right\rangle$ such that $\mathbf{S}=\mathbf{S}^{\prime}$ and $|\mathbf{S}| \in 2 \mathbf{Z}^{+}$is considered, an additional projection must be taken into account, due to the $\psi$ labels, leading to

$$
\frac{1}{2^{|\mathbf{S}|}} \sum_{\lambda, \mu}^{s r} \sum_{\varepsilon_{1}, \ldots, \varepsilon_{r}=0}^{1}\left(\prod_{i=1}^{r} N_{L_{j}, \tilde{L}_{j}}^{\left|\varepsilon_{j} k_{j}-l_{j}\right|}\right) \delta_{\sum_{i \in \mathbf{S}} \varepsilon_{i}=\frac{1}{2}\left(1-\psi \psi^{\prime}\right)}^{(2)} \delta_{\sum_{i} \varepsilon_{i}=1+\frac{s_{0}}{2}}^{(2)} \chi_{\mu}^{\lambda}
$$

On the other hand, the massless states in the bosonic Möbius strip amplitude are given by

$$
-\frac{1}{2 N_{\mathbf{S}}} \prod_{k<l}(-1)^{\rho_{k} \rho_{l}} \delta_{\sum \rho_{j}+1+\frac{s_{0}}{2}, \Sigma \omega_{j}}^{(2)}(-1)^{\sum \omega_{j} \frac{s_{0}}{2}} e^{i \frac{\pi}{2} \sum_{j} \omega_{j}\left(m_{j}-2 L_{j}+\varepsilon_{j}\left(k_{j}+2\right)\right)}(-1)^{\varepsilon_{j}} N_{L_{j} L_{j}}^{\left|\varepsilon_{j} k_{j}-l_{j}\right|} \hat{\chi}_{\mu}^{\lambda}
$$

where $\rho_{j}=\frac{s_{0}}{2}+1+\varepsilon_{j}+\sum \omega_{j}$.

In particular, we see the vector $\left(s_{0}=2\right)$ has the sign

$$
-\frac{1}{N_{\mathbf{S}}}(-1)^{\sum \omega_{j}}(-1)^{\sum \omega_{j} L_{j}} \sum_{\varepsilon_{1}, \ldots, \mathcal{\varepsilon}_{r}=0}^{1} \prod_{k<l}(-1)^{\varepsilon_{k} \varepsilon_{l}} \prod(-1)^{\omega_{j} \varepsilon_{j} \frac{k_{j}+2}{2}} \delta_{\varepsilon_{j} L_{j}, \varepsilon_{j} \frac{k_{j}}{2}} \delta_{\sum \varepsilon_{j}, \sum \omega_{j}}
$$

A plus (minus) sign indicates a symplectic (orthogonal) gauge group while a zero leads to a unitary gauge group. In a similar manner, the gauge group representations in which matter states transform, can be identified (an example is given in next section).

The tadpole cancellation conditions can be easily read from the expressions for the crosscap (3.2) and boundary states (3.4). They take the general form $\operatorname{Tad}_{D}(\lambda, \mu)-8 \operatorname{Tad}_{O}(\lambda, \mu)=0$. For the massless fields $(2,0)(0,0,0)^{6}$ and $(0,0) \prod_{j}\left(l_{j}, l_{j}, 0\right)$ the NS-NS tadpoles of the orientifold plane read

$$
\begin{aligned}
\operatorname{Tad}_{O}(\lambda, \mu)_{B}= & \sum_{v_{0}=0}^{\frac{K}{2}-1} \sum_{\varepsilon_{1}, \ldots, \varepsilon_{r}=0}^{1}\left(\prod_{k<l}(-1)^{\varepsilon_{k} \varepsilon_{l}}\right)(-1)^{v_{0} \sum \varepsilon_{j}} \delta_{s_{0} / 2,1+\sum \varepsilon_{j}+\sum \omega_{j}}^{(2)}(-1)^{\sum \omega_{j}\left(s_{0} / 2\right)}(-1)^{\Delta_{j}\left(1-\varepsilon_{j}\right)} \\
& \prod_{j=1}^{r}\left(\sin \left[\frac{1}{2}\left(l_{j}, \varepsilon_{j} k_{j}\right)\right] \delta_{l_{j}+\left(1-\varepsilon_{j}\right) k_{j}, 0}^{(2)} \delta_{m_{j}^{\prime}, 2 v_{0}+\left(1-\varepsilon_{j}+\omega_{j}\right)\left(k_{j}+2\right)}^{\left(2 k_{j}+4\right)}(-1)^{\varepsilon_{j} \frac{m_{j}}{2}}\right)
\end{aligned}
$$


Also, collecting all terms from the boundary states and their $\Omega_{\Delta_{j}, \omega, \omega_{\alpha}}$ images, we obtain, for their massless tadpoles

$$
\operatorname{Tad}_{D}(\lambda, \mu)=\sum_{a=1}^{N} \frac{N_{a}}{N_{\mathbf{S}}} \cos \left[\pi \sum_{j} \frac{m_{j}\left(M_{j}^{a}-\Delta_{j}\right)}{k_{j}+2}\right] \prod_{j} \sin \left(l_{j}, L_{j}^{a}\right) .
$$

These expressions are valid up to common phases. We have also renormalized the tadpole equations by introducing the factor $N_{\mathbf{S}}$ so that the Chan-Paton factors $N_{a}$ truly represent the multiplicity of elementary D-branes.

\section{$3.1(6)^{2}(2)\left(0^{3}\right)$ model}

We exemplify the construction presented in the preceeding section for the specific Gepner model $(6)^{2}(2)\left(0^{3}\right)$. We will later consider this example to discuss model building in four dimensions.

The gauge groups and massless spectrum is obtained from Eqs. (3.8) and (3.9). For instance, we see that brane $\mathbf{L}_{\mathbf{1 0}}=3310^{3}$ is short, with $|\mathbf{S}|=6$. Thus, for the vector $\left(s_{0}=2\right)$, a non vanishing contribution in (3.8) implies $\delta_{\sum_{i} \varepsilon_{i} \frac{1}{2}\left(1-\psi \psi^{\prime}\right)}^{(2)} \delta_{\sum_{i} \varepsilon_{i}=0}^{(2)} \neq 0$, namely $\psi=\psi^{\prime}$. Moreover, for such choice of $\varepsilon_{i}^{\prime} s$ we see that (3.9) vanishes thus leading to the unitary group. In a similar way, for the scalars $\left(s_{0}=0\right)$ the states propagating between a boundary state and its orientifold image are selected, $\psi=-\psi^{\prime}$. Möbius amplitude (3.9) is non vanishing in this case and produces a minus sign thus leading to antisymmetric representations.

The tadpole equations $(3.11,3.12)$ for this set of branes reads

$$
\begin{array}{r}
N_{2}+2 N_{3}+N_{4}+N_{5}+2 N_{6}+N_{7}+2 N_{8}+N_{9}+2 N_{10}+N_{11}+3 N_{12}=16 \\
N_{1}+2 N_{2}+2 N_{3}+2 N_{4}+N_{5}+3 N_{6}+2 N_{8}+2 N_{9}+2 N_{10}+2 N_{11}+4 N_{12}=24
\end{array}
$$

States propagating between branes can be easily computed from (3.7) and (3.8). Two tensor multiplets are found in the internal sector (see for instance [3]). It can be checked that all gauge and gravitational anomalies cancel.

At this point it may be instructive and useful for our subsequent calculations to illustrate this in a detailed example containing only $\left(\mathbf{L}_{\mathbf{1}}, \mathbf{L}_{\mathbf{6}}, \mathbf{L}_{\mathbf{1 0}}\right)$ states.

The tadpole equations for the reduced set of D-branes lead to

$$
N_{10}=8-N_{6}=N_{1}
$$

The gauge group is $\operatorname{Sp}\left(N_{1}\right) \times S p\left(N_{6}\right) \times U\left(N_{10}\right)$ with matter hypermultiplets in

$$
\begin{aligned}
& 3[(1,1, \boxminus)+(1,1, \bar{\boxminus})]+7(1, \boxminus, 1)+(1, \square, 1) \\
+ & \left(N_{1}, N_{6}, 1\right)+\left(N_{1}, 1, N_{10}\right)+\left(N_{1}, 1, \bar{N}_{10}\right)+3\left[\left(1, N_{6}, N_{10}\right)+\left(1, N_{6}, \bar{N}_{10}\right)\right]
\end{aligned}
$$

It is easy to check that this spectrum (plus a closed sector containing two tensor multiplets and nineteen hypermultiplets) leads to vanishing of gauge and gravitational anomalies if tadpole equations (3.14) are satisfied. 


\section{Orbifolding (Gepner model $)^{c=6} \times \mathbf{T}^{2}$}

Orbifolds of Gepner models are also easily implemented in the language of boundary and crosscap states. The internal sector described by $(\text { Gepner model })^{c=6} \times \mathbf{T}^{2}$ has a discrete symmetry acting on fields in the following way

$$
g: Z \rightarrow e^{2 \pi i v} Z \quad g: \Phi_{m_{i} s_{i}}^{l_{i}} \rightarrow e^{2 \pi i \frac{m_{i} \gamma_{i}}{k_{i}+2}} \Phi_{m_{i} s_{i}}^{l_{i}} i=1, \ldots, r
$$

where $Z=X^{4}+i X^{5}$ denotes the complex coordinate on $\mathbf{T}^{2}$ and $\left(v ; \gamma_{i}\right)$ are labels for the generator $\hat{g} \in G$. For a torus with symmetry $\mathbf{Z}_{\mathbf{N}}$ we have $N v \in \mathbf{Z}$. The labels $\left(v ; \gamma_{i}\right)$ are conveniently encoded in terms of a simple current vector $j$

$$
j=\left(0, v ; 2 \gamma_{1}, \ldots, 2 \gamma_{r} ; 0, \ldots, 0\right) .
$$

which satisfies $2 \beta_{0} \bullet j \in \mathbf{Z}$ or in components

$$
-\frac{v}{2}+\sum \frac{\gamma_{i}}{k_{i}+2}=0 \quad \bmod \mathbf{Z}
$$

As it is well known, twisted sectors must be included in order to ensure the modular invariance of the torus partition function. As a consequence, new tadpoles are expected to appear, in the transverse channel, due to RR fields propagating in the twisted sectors. Thus, the boundary states required to cancel the tadpoles include the RR fields in the twisted sector of the theory.

Interestingly enough, it is possible to rewrite the projection by simple currents in such a way that its relation to the usual orbifolds of toroidal manifolds is much more evident. To see this we recall that open string states formally read

$$
\left|\Phi_{k} ; i, j\right\rangle \lambda_{j i}^{k}
$$

where $\lambda^{k}$ encodes the gauge group representation into which the state $\Phi_{k}$ transforms. For instance, if the state $\Phi_{0}$ corresponds to gauge bosons, $\lambda^{0}$ represents gauge group $G$ generators ${ }^{2}$.

Let us assume that such Chan-Paton factors have been determined already and that we further act on string states with a generator $\theta$ of a $Z_{N}$ symmetry group. Such action which manifests as a phase $e^{2 \pi i \frac{\gamma_{i} m_{i}}{k_{i}+2}}$ on world sheet field $\Phi_{k}$ should, in principle, be accompanied by corresponding representation of group action such that

$$
\hat{\theta}\left|\Phi_{k} ; i, j\right\rangle \lambda_{j i}=\gamma_{i i^{\prime}}\left|\hat{\theta} \Phi_{k} ; i^{\prime}, j^{\prime}\right\rangle \gamma_{j^{\prime} j} \lambda_{j i}=e^{2 \pi i \frac{\gamma_{i} m_{i}}{k_{i}+2}}\left(\gamma^{-1} \lambda \gamma\right)_{j^{\prime} i^{\prime}}\left|\Phi_{k} ; i^{\prime}, j^{\prime}\right\rangle
$$

Therefore, invariance under such action requires

$$
e^{2 \pi i \frac{\gamma_{i} m_{i}}{k_{i}+2}} \gamma^{-1} \lambda^{k} \gamma=\lambda^{k}
$$

By following the same steps as in Ref. [23], we can represent $Z_{N}$ Chan-Paton twist in terms of Cartan generators as $\gamma=e^{2 \pi i V H}$ where $V$ is a "shift" eigenvalues vector of the generic form

$$
V=\frac{1}{N}\left(0^{N_{0}}, 1^{N_{1}}, \ldots,(N-1)^{N_{N-1}}\right)
$$

\footnotetext{
${ }^{2}$ Which generically will be a product of unitary, orthogonal and symplectic groups.
} 
(ensuring $\gamma^{N}=1$ ) and Cartan generators are represented by $2 \times 2 \sigma_{3}$ submatrices.

On this basis, projection equation (4.5) reduces to the simple condition

$$
\rho_{k} V=\frac{\gamma_{i} m_{i}}{k_{i}+2}+\frac{v s_{-1}}{2}
$$

where $\rho_{k}$ is the weight vector associated to the corresponding $\lambda^{k}$ representation.

Recall that in the orientifold theory we must introduce a boundary state and its image under $\Omega$.

Tadpole conditions can be generalized for orbifolded hybrid models $T^{2} \times$ Gepner in the following way [6]

$$
\begin{gathered}
D_{\mu}^{\lambda}\left(\operatorname{Tr} \gamma_{N, 2 x}+\sqrt{f} \operatorname{Tr} \gamma_{D, 2 x, I}\right)+O_{\mu}^{\lambda} \cos \pi x v=0 \\
D_{\mu}^{\lambda}\left(\operatorname{Tr} \gamma_{N, 2 x+1}+\sqrt{f} \operatorname{Tr} \gamma_{D, 2 x+1, I}\right)=0
\end{gathered}
$$

for all states $(\lambda, \mu)$ such that $\chi_{\mu+2 x \Gamma}^{\lambda}$ is massless.

Here $O_{\mu}^{\lambda}$ is the orientifold charge we have in six dimensions for the state $(\lambda, \mu)$ while the factor $f=4 \sin ^{2} \pi x v$ is a non-trivial contribution from the fixed points in the complex torus $\mathrm{T}^{2}$ in the NN sector. The labels N and D are used to distinguish between D-branes with Neumann and Dirichlet boundary conditions in the torus $T^{2}$.

\section{A MSSM example}

As an illustration of the general techniques discussed above we concentrate here on a $\mathbf{Z}_{\mathbf{4}}$ modding of the $\left[(6)^{2}(2)(0)\right]^{c=6} \times T^{2}$ model $^{3}$. Let us notice that inspection of allowed internal states indicates that only 3 massless chiral $\left(l_{i}=m_{i}, s_{i}=0\right)$ states, namely those such $\left(m_{1}, m_{2}, m_{3}, m_{4}\right)=$ $(3,3,1,0),(5,1,1,0),(1,5,1,0)$, do propagate between brane $\mathbf{L}_{\mathbf{1 0}}$ (with a $U\left(N_{10}\right)$ gauge group living on its worldvolume) and $\mathbf{L}_{\mathbf{6}}$ (with an $S p\left(N_{6}\right)$ gauge group). Therefore, an internal modding of the form $\Gamma=(0,0,1,0)$ acting on the Gepner model will allow such states to remain in the spectrum and, by appropriately embedding it as a twist $\gamma^{10}, \gamma^{6}$ on the D-brane sector, the original $U\left(N_{10}\right) \times S p\left(N_{6}\right)$ gauge group could be broken into a Standard-like model with 3 generations. Moreover, in order to have $\mathscr{N}=1$ supersymmetry in four dimensions, we must accompany this modding with a $\mathbf{Z}_{\mathbf{2}}$ modding on $T^{2}$, namely $v_{3}=1 / 2$, so as to satisfy Eq.(4.3). Thus, our starting point is

$$
\Gamma=(0,0,1,0)\left(\frac{1}{2}\right) \otimes \gamma^{a}
$$

Note that the actual internal modding (see (4.1)) is $\gamma_{i} /\left(k_{i}+2\right)$ so it represents a $Z_{4}$ action.

As we stressed in the previous section, performing a $\mathbf{Z}_{\mathbf{2}}$ modding on the torus will require the introduction of a new set of branes having Dirichlet boundary conditions on the open string ends living on $T^{2}$. We quote them with an index $D$ while introducing an index $N$ to label the original branes with Neumann conditions on the third complex coordinate $Z$. We will refer to them as $D_{Z}$ and $N_{Z}$ branes respectively. Besides the untwisted tadpole equations 3.13, several new equations must now be solved (see [7] due to the twisting, according to Eq.(4.8,4.9).

\footnotetext{
${ }^{3}$ We will write the internal sector in terms of four theories in what follows.
} 
As mentioned, $\mathbf{L}_{\mathbf{1 0}}$ and $\mathbf{L}_{\mathbf{6}}$ constitute the basic branes which, after splitting under modding action, will give rise to our model. It is interesting to remark that, both boundary states can be placed on the same NN sector, or either $\mathbf{L}_{\mathbf{1 0}}$ in NN sector and $\mathbf{L}_{\mathbf{6}}$ in the DD sector (or viceversa) or both in the DD sector. The basic features, discussed below, will be independent of the sector choice. However phenomenological details will be different, mainly due to the extra branes that must be added to satisfy RR tadpole cancellation.

We choose two (minimal) $N_{10}=N_{6}=4$ stacks of $\mathbf{L}_{\mathbf{6}}$ and $\mathbf{L}_{\mathbf{1 0}}$ branes to start with a $U(4) \times$ $S p(4)$ gauge group. The modding $\Gamma$ in ( 5.1) is embedded as twists $\gamma_{6}$ and $\gamma_{10}$, on each respective stack, as

$$
\begin{aligned}
\gamma_{6} \rightarrow V_{6} & =\frac{1}{4}(0,2) \\
\gamma_{10} \rightarrow V_{10} & =\frac{1}{4}(1,1,1,3)
\end{aligned}
$$

For the vector $\Gamma . \mu=0$ and therefore from (4.7) we find

$$
\begin{aligned}
U(4) & \rightarrow S U(3) \times U(1)^{2} \\
S p(4) & \rightarrow S U(2) \times S U(2)
\end{aligned}
$$

where $S p(2) \equiv S U(2)$. Thus, a LR symmetric-like model group is obtained.

Moreover, the correct LR spectrum with 3 massless generations is found. Namely, massless chiral states propagating in between $\mathbf{L}_{\mathbf{1 0}}-\mathbf{L}_{\mathbf{6}}$ (these are $\left.(0 ; 3310 ; 0), \quad(0 ; 15100), \quad(0 ; 5110 ; 0)\right)$ satisfy $\Gamma \mu=\frac{1}{4}$ and therefore we find the spectrum representations under $S U(3) \times S U(2)_{L} \times S U(2)_{R} \times$ $Q_{B-L}$ to be

$$
3\left[(3,2,1)_{\frac{1}{3}}+(\overline{3}, 1,2)_{-\frac{1}{3}}+(1,1,2)_{1}+(1,2,1)_{-1}\right]
$$

where the subindex indicates the charge eigenvalue of

$$
Q_{B-L}=\frac{1}{3} Q_{3}+Q
$$

$Q_{3}$ being the generator of the $U(1)$ in $U(3)$ and $Q$ the other $U(1)$ generator in (5.5).

Actually, it is possible to establish a correspondence with an intersecting brane model picture in toroidal manifolds (see for instance [11]). Namely, under the action of $\gamma_{10}$ and $\gamma_{6}$, boundary states $\mathbf{L}_{\mathbf{1 0}}$ and $\mathbf{L}_{\mathbf{6}}$ intersecting at a six dimensional manifold, split into four stacks of boundary states as

$$
\begin{aligned}
& \mathbf{L}_{\mathbf{1 0}}[U(4)] \rightarrow \mathbf{L}_{\mathbf{1 0}}^{\mathbf{a}}[U(3)]+\mathbf{L}_{\mathbf{1 0}}^{\mathbf{d}}[U(1)] \\
& \mathbf{L}_{\mathbf{6}}[S p(4)] \rightarrow \mathbf{L}_{\mathbf{6}}^{\mathbf{b}}[S p(2)]+\mathbf{L}_{\mathbf{6}}^{\mathbf{c}}[S p(2)]
\end{aligned}
$$

where we have indicated in brackets the gauge group living on the corresponding brane. Thus, boundary states $\mathbf{L}_{\mathbf{1 0}}^{\mathbf{a}}, \mathbf{L}_{\mathbf{1 0}}^{\mathbf{d}}, \mathbf{L}_{\mathbf{6}}^{\mathbf{b}}, \mathbf{L}_{\mathbf{6}}^{\mathbf{c}}$ do match with the basic branes $a, b, c, d$ arising in intersecting brane models on toroidal constructions [11, 24].

Thus, drawing boundary states as lines and interpreting multiplicities as intersection numbers we are lead to a graphic representation as the one given in left side of Figure 1. 

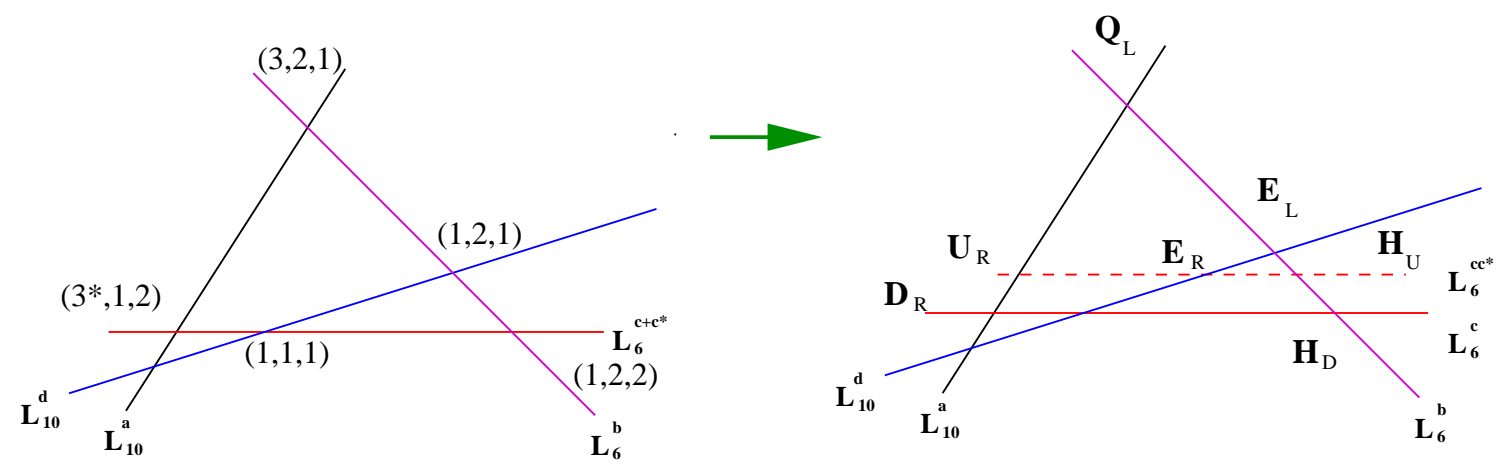

Figure 1: By separating $\mathbf{L}_{\mathbf{6}}^{\mathbf{c}+\mathbf{c} *} \rightarrow \mathbf{L}_{\mathbf{6}}^{\mathbf{c}}+\mathbf{L}_{\mathbf{6}}^{\mathbf{c} *}$ away from the orientifold point breaking from LR to MSSM with 3 righthanded neutrini is achieved

Besides states propagating between different branes we must consider states along the same type of branes. They lead to vector like matter.

Interestingly enough, massless states (4020), (0420) and (2220) do propagate in $\mathbf{L}_{\mathbf{6}}-\mathbf{L}_{\mathbf{6}}$ sector. They satisfy $\Gamma . \mu=\frac{1}{2}$ and thus, together with (1)(0000), descending from the six dimensional vector, lead to $9(1,2,2)_{0}$ candidates to LR Higgses.

Branes $c$ and its image $c *$ are placed here on top of each other and on top of an orientifold point (leading to $S p(2)$ ). Since such branes are parallel in the torus, following similar steps as discussed [12], we can think into separating them away from the orientifold point in the torus. Thus, $S U(2)_{R} \rightarrow U(1)_{c}$ where $U(1)_{c}$ charges are given by $T_{R}^{3}$ eigenvalues. Therfore, by introducing the weak hypercharge $Y=-T_{R}^{3}+\frac{1}{2} Q_{B-L}$ we find that the original LR symmetric model breaks down to $S U(3) \times S U(2)_{L} \times U(1)_{Y}$ MSSM with three chiral generations

$$
3\left[(3,2,1)_{\frac{1}{6}}+(\overline{3}, 1)_{-\frac{2}{3}}+(\overline{3}, 1)_{\frac{1}{3}}+(1,1)_{\frac{1}{2}}+(1,2)_{-\frac{1}{2}}+(1,1)_{0}\right]
$$

including three right handed neutrini. Moreover, $L R$ chiral states $(1,2,2)_{0}$ decompose into $(1,2)_{-1 / 2}+$ $(1,2)_{1 / 2}$ with the correct MSSM Higgs charges. It can be checked that, non-anomalous, hypercharge remains massless in the model under consideration [7]. A pictorial representation is presented in Figure 1.

Besides these basic boundary states leading to the MSSM structure, additional stacks of branes must be added in order to satisfy tadpole cancellation equations. Different choices are possible and each of them will give rise to particular phenomenological features. A simple explicit example can be found Ref. [7].

Some general remarks about Yukawa couplings can be advanced. As a general observation notice that a Yukawa coupling will have the form

$$
Y_{i j k} \Phi_{b a}^{i} \Phi_{a c}^{j} \Phi_{c b}^{k}
$$

where $\Phi_{a b}$ is the chiral superfield insertion connecting boundaries $a$ and $b$ and $i, j, k$ refers to internal CFT labels. Such a term should be a singlet of the gauge group and invariant under $\Gamma$ modding. Moreover, it must be allowed by the fusion rules of the internal conformal field theory [25, 21]. Namely, $Y_{i j k} \propto\langle i j k\rangle \propto \mathscr{N}_{i j}^{k}$ 
For instance, couplings like

$$
\left.[(\underline{51} 10)](3,2,1)_{1 / 3}^{a b} \times[(3310))\right](\overline{3}, 1,2)_{-1 / 3}^{a c}[(2220)](1,2,2)_{0}^{b c}
$$

(where we have indicated the internal charges in brackets) are non vanishing and lead to degenerate masses for two quark generations. Fusion rules forbid masses for the first quark generation. A similar result is obtained for lepton masses since the same internal states are involved for leptonic Yuakawa couplings. The general pattern is very similar (the number of Higgses is different) to the one found in Ref. [26] in the context of branes at singularities.

Actually, (see i.e. [26], the full picture of mass structures becomes more complicated due, for instance, to the presence of Yukawa couplings of quarks with colored triplets present at other intersections. Notice also that, from the 9 candidates to be interpreted as Higgs particles coming from $\mathbf{L}_{\mathbf{6}}-\mathbf{L}_{\mathbf{6}}$ sector, only those with CFT quantum numbers (2220) are allowed in Yukawa couplings. For all of them, on the other hand, mass term like couplings are allowed. Thus, we can imagine a scenario where some of the $(1,2,2)$ multiplets become very massive.

In the example discussed above the basic branes $\mathbf{L}_{\mathbf{1 0}}$, containing strong group, and $\mathbf{L}_{\mathbf{6}}$, where $S U(2)_{L} \times S U(2)_{R}$ lives, were placed in the same NN sector. However, other schemes, which might be useful future phenomenological applications, are also possible. For instance, consistent models where part of the spectrum containing the $S U(2)_{L} \times S U(2)_{R}$ gauge theory is placed on the branes in the DD sector can be built.

An interesting feature of the hybrid construction is that lowering of the string scale [27] could be achieved by considering large extra dimensions in the $T^{2}$ torus, transverse to the whole configuration of intersecting boundary states.

Indeed, in the present examples of the type Gepner $\times T^{2} / \mathbf{Z}_{\mathbf{N}}$, boundary states would correspond to branes wrapping cycles on $K_{3}$ and stuck at a $\mathbf{C} / \mathbf{Z}_{\mathbf{N}}$ singularity. Thus, if we denote by $V_{4}$ the volume of the Gepner piece, which should be of the order of the string scale $V_{4} \propto 1 / M_{s}^{4}$, and by $V_{2}$ that of two dimensional manifold. Then we expect the Planck scale, after dimensional reduction to four dimensions, to be

$$
M_{\text {Planck }}=\frac{2}{\lambda} M_{s}^{4} \sqrt{V_{4} V_{2}}
$$

where $\lambda$ is the string coupling. Therefore the string scale $M_{s}$ can be lowered by choosing the volume $V_{2}\left(V_{2}=\frac{M_{\text {Planch }}^{2} \lambda^{2}}{4 M_{s}^{4}}\right)$ sufficiently large. Recall that the models constructed here are fully supersymmetric and though lowering the scale could be phenomenologically attractive in some cases it is not as compelling as in non supersymmetric models.

Presumably, having these large extra dimension could allow for the introduction of dilute fluxes in a supergravity limit of some of these hybrid construction [28].

\section{Acknowledgments}

We are grateful to the organizers of Fifth International Conference on Mathematical Methods in Physics for the invitation to present this talk and for kind hospitality.

\section{References}

[1] R. Blumenhagen and A. Wisskirchen, Spectra of 4D, $N=1$ type I string vacua on non-toroidal CY threefolds, Phys. Lett. B438 (1998) 52, [hep-th/9806131]. S. Govindarajan and J. Majumder, Crosscaps in Gepner models and type IIA orientifolds, JHEP 0402 (2004) 026, [hep-th/ 0306257 ]. 
[2] R. Blumenhagen, Supersymmetric orientifolds of Gepner models JHEP 0311, 055 (2003), [hep-th/0310244].

R. Blumenhagen and T. Weigand, A Note on Partition Function of Gepner Model Orientifolds Phys.Lett. B591 (2004) 161-169, [hep-th/ 0403299 ].

[3] G. Aldazabal, E. C. Andres, M. Leston and C. Nunez,Type IIB orientifolds on Gepner points JHEP 0309, 067 (2003), [hep-th/0307183].

[4] I. Brunner, K. Hori, K. Hosomichi and J. Walcher,Orientifolds of Gepner models, [hep-th/0401137].

[5] T. P. T. Dijkstra, L. R. Huiszoon and A. N. Schellekens, Chiral supersymmetric standard model spectra from orientifolds of Gepner models, Phys. Lett. B 609, 408 (2005), [hep-th/ 0403196. T.P.T. Dijkstra, L.R. Huiszoon, A.N. Schellekens, Supersymmetric Standard Model Spectra from RCFT Orientifolds, Nucl.Phys.B710:3-57,2005, [hep-th/0411129].

[6] G. Aldazabal, E. C. Andres and J. E. Juknevich, Particle models from orientifolds at Gepner-orbifold points, JHEP 0405, 054 (2004), [hep-th / 0403262 ].

[7] G. Aldazabal, E. Andres and J. E. Juknevich, On Susy Standard-like models from orbifolds of D=6 Gepner orientifolds, JHEP07(2006)039, [hep-th/ 0603217$].$

[8] D. Gepner, Lectures On N=2 String Theory, Lectures at Spring School on Superstrings, Trieste, Italy, Apr 3-14, 1989.

[9] J. Fuchs, A. Klemm, C. Scheich and M. G. Schmidt, Spectra And Symmetries Of Gepner Models Compared To Calabi-Yau Compactifications, Annals Phys. 204, 1 (1990). J. Fuchs, A. Klemm, C. Scheich and M. G. Schmidt, Gepner Models With Arbitrary Affine Invariants And The Associated Calabi-Yau Spaces, Phys. Lett. B 232, 317 (1989).

A. Font, L. E. Ibanez, M. Mondragon, F. Quevedo and G. G. Ross, (0,2) Heterotic String Compactifications From N=2 Superconformal Theories, Phys. Lett. B 227, 34 (1989).

D. Gepner, String Theory On Calabi-Yau Manifolds: The Three Generations Case, [hep-th/9301089].

C. Angelantonj, M. Bianchi, G. Pradisi, A. Sagnotti and Y. Stanev, Comments on Gepner models and Type I vacua in string theory, Phys. Lett. $B 387$ (1996) 743, [hep-th/960722].

[10] J. Fuchs, A. Klemm, C. Scheich and M. G. Schmidt, Spectra And Symmetries Of Gepner Models Compared To Calabi-Yau Compactifications, Annals Phys. 204, 1 (1990).

[11] L. E. Ibanez, F. Marchesano and R. Rabadan, Getting just the standard model at intersecting branes, JHEP 0111 (2001) 002, [hep-th/ 0105155$]$. D. Cremades, L. E. Ibanez and F. Marchesano, Intersecting brane models of particle physics and the Higgs mechanism, JHEP 0207, 022 (2002) [hep-th/0203160].

[12] D. Cremades, L. E. Ibanez and F. Marchesano, Yukawa couplings in intersecting D-brane models, JHEP 0307, 038 (2003), [hep-th/ 0302105$].$

[13] R. Blumenhagen, L. Goerlich, B. Kors, D. Lust, Noncommutative compactifications of type I strings on tori with magnetic background flux. JHEP 0010:006, 2000, [hep-th / 0007024 ].

R. Blumenhagen, B. Kors, D. Lust; Type I strings with F flux and B flux. JHEP 0102:030, 2001, [hep-th/0012156].

M. Berkooz, M. R. Douglas, R. G. Leigh, Branes at angles, Nucl.Phys. B480:265-278,1996, [hep-th/9606139]. 
G. Aldazabal, S. Franco, Luis E. Ibanez, R. Rabadan, A.M. Uranga, Intersecting brane worlds. JHEP 0102:047,2001, [hep-ph/0011132].

A. M. Uranga, Chiral four dimensional string compactifications with intersecting D-branes.

Class.Quant.Grav. 20:S373-S394,2003, [hep-th/0301032].

[14] J. Fuchs, P. Kaste, W. Lerche, C. A. Lutken, C. Schweigert and J. Walcher, Boundary fixed points, enhanced gauge symmetry and singular bundles on K3, Nucl. Phys. B 598 (2001) 57,

[hep-th/0007145].

[15] C. Angelantonj, A. Sagnotti, Open Strings Phys.Rept. 371 (2002) 1-150; Erratum-ibid. 376 (2003) 339-405, [hep-th/0204089].

[16] N. Ishibashi, The boundary and crosscap states in conformal field theories, Mod. Phys. Lett. A4 (1989) 251; N. Ishibashi, T. Onogi, Conformal field theories on surfaces with boundaries and crosscaps, Mod. Phys. Lett. A4 (1989) 161. H. Ooguri, Y. Oz and Z. Yin, D-branes on Calabi-Yau spaces and their mirrors, Nucl. Phys. B477 (1996) 407, [hep-th/9606112].

[17] J. Polchinski, Dirichlet-Branes and Ramond-Ramond Charges, Phys. Rev. Lett. 75, 4724 (1995), [hep-th/9510017]. E. G. Gimon, J. Polchinski, Consistency conditions for orientifolds and D manifolds, Phys.Rev.D54:1667-1676,1996; [hep-th/9601038].

J. Polchinski, S. Chaudhuri and C. V. Johnson, Notes on D-branes, [hep-th/9602052].

[18] M. Bianchi and A. Sagnotti,On The Systematics Of Open String Theories, Phys. Lett. B247 (1990) 517.

[19] V. Braun and S. Schafer-Nameki, D-brane charges in Gepner models, [hep-th/0511100].

[20] A. Recknagel and V. Schomerus, D-branes in Gepner modelsB Nucl. Phys. B531 (1998) 185, [hep-th/9712186].

[21] I. Brunner and V. Schomerus, On superpotentials for D-branes in Gepner models, JHEP 0010, 016 (2000), [hep-th/0008194].

[22] P. Di Francesco, P. Mathieu and D. Senechal, "Conformal field theory," 1997. New York, USA: Springer (1997) $890 \mathrm{p}$.

[23] G. Aldazabal, A. Font, L. E. Ibanez and G. Violero, D =4, $N=1$, type IIB orientifolds, Nucl. Phys. B 536 (1998) 29, [hep-th/9804026].

[24] F. Gmeiner, R. Blumenhagen, G. Honecker, D. Lust and T. Weigand, One in a billion: MSSM-like D-brane statistics, JHEP 0601, 004 (2006), [hep-th/ 0510170$].$

[25] J. Distler and B. R. Greene, Some Exact Results On The Superpotential From Calabi-Yau Compactifications, Nucl. Phys. B 309, 295 (1988).

[26] G. Aldazabal, L. E. Ibanez and F. Quevedo, A D-brane alternative to the MSSM, JHEP 0002, 015 (2000), [hep-ph/0001083].

[27] N. Arkani-Hamed, S. Dimopoulos and G. R. Dvali, “The hierarchy problem and new dimensions at a millimeter,” Phys. Lett. B 429, 263 (1998), [hep-ph/ 9803315 ]. I. Antoniadis, N. Arkani-Hamed, S. Dimopoulos and G. R. Dvali, “New dimensions at a millimeter to a Fermi and superstrings at a TeV,” Phys. Lett. B 436, 257 (1998), [hep-ph/ 9804398 ].

D. Cremades, L. E. Ibanez and F. Marchesano, Standard model at intersecting D5-Branes: lowering the string scale, Nucl. Phys. B643 (2002) 93, [hep-th/ 0205074 ]. C. Kokorelis, Exact standard model structures from intersecting D5-branes," Nucl. Phys. B 677 (2004) 115,[hep-th/ 0207234 ].

[28] M. Grana, Flux compactifications in string theory: A comprehensive review, Phys. Rept. 423, 91 (2006), [hep-th/0509003]. 